Case Report

\title{
Nasopharyngeal Branchial Cyst, a Rare Presentation
}

\author{
Wael Al Juraibi \\ Department of Otolaryngology \& Head and Neck Surgery, Al Hayat National Hospital, Jazan, Saudi Arabia
}

Email address

drwael39@yahoo.com,drwael39@gmail.com

\section{To cite this article:}

Wael Al Juraibi. Nasopharyngeal Branchial Cyst, a Rare Presentation. Journal of Surgery. Special Issue: Surgical Infections and Sepsis. Vol. 4, No. 3-1, 2016, pp. 25-28. doi: 10.11648/j.js.s.2016040301.15

Received: March 14, 2016; Accepted: March 16, 2016; Published: April 18, 2016

\begin{abstract}
Introduction: Branchial cleft cysts are congenital developmental defects of which second branchial anomalies are the most common type. Most of these anomalies present as a lateral neck mass along anterior border of sternocliedomastoid muscle. Careful examination and proper intervention is needed in some of these cases to avoid unwanted complications or even emergencies. Case presentation: A 5 years old boy was brought by his parents with history of dysphagia and snoring for about one month. There was no other associated history of sore throat or shortness of breath or oral bleeding. During fiberoptic examination we found a pedunculated left nasopharyngeal mass. CT imaging showed a left nasopharyngeal hypodense lesion with no vascular or bony invasion. Excision of the cyst was done via combined transoral/transnasal endoscopic approach. Follow up after eight months showed no evidence of recurrence. Conclusion: Second branchial cleft cysts presenting in the nasopharynx are considered rare presentations of the disease and other differential diagnosis should be always brought in mind. Fiberoptic examination of such cases is mandatory to rule out laryngeal involvement and to predict the extension of the cyst for surgical intervention. Surgical excision through combined transoral/transnasal endoscopic including the tract ligation is the treatment of choice to prevent recurrence and to minimize the occurrence of possible secondary infection of the cyst.
\end{abstract}

Keywords: Branchial Cleft, Nasopharyngeal Cyst, Pediatric, Transoral Excision

\section{Introduction}

Branchial cleft cysts are congenital developmental defects of which second branchial anomalies are the most common type. It can occur anywhere along the line from the tonsillar fossa to the supraclavicular area of the neck [1]. Branchial anomalies account for about $17 \%$ of pediatric neck masses [2]. Most of these anomalies present as a lateral neck mass along anterior border of sternocliedomastoid muscle. Other rare presentations have been reported such as nasopharyngeal cyst, parapharyngeal cysts and even as a posterior neck mass [3-5]. Careful examination and proper intervention is needed in some of these cases to avoid unwanted complications or even emergencies. Elective surgical excision has been the treatment of choice for all branchial cleft cysts. Abscesses should first be treated with incision and drainage. The cyst should then be removed, with its tract traced to its origin in the aerodigestive system. Recurrence can occur in as many as $22 \%$ of patients who have had previous surgery [6]. We are presenting a case of $2 \mathrm{nd}$ branchial cleft cysts presenting in the nasopharynx.

\section{Case Presentation}

A 5 years old boy was brought by his parents with history of dysphagia and snoring for about one month. There was no other associated history of sore throat or shortness of breath or oral bleeding. No history of fever or weight loss was noticed. Past medical and family history were negative. Throat examination showed a swelling behind soft palate. During Fiberoptic examination we found a pedunculated left nasopharyngeal mass measuring about $1.5 * 2 \mathrm{~cm}$ with normal over lying mucosa \{figure1\}. The mass looked cystic and compressible with no extension into hypopharynx and larynx. There was no evidence of infection. Otological examination showed no middle ear effusion, indicating absence of Eustachian tube obstruction. CT imaging \{figures $2 \& 3$ \} showed a left nasopharyngeal hypodense lesion with no vascular or bony invasion. Excision of the cyst was done via combined transoral/transnasal endoscopic approach using cold method and electrocautery for hemostasis, with ETT in 
place. Histopathological examination revealed thick walled cyst lined by respiratory epithelium with nodular lymphoid infiltrate consistent with branchial cleft cyst with no evidence of malignancy or atypia \{figures $4 \& 5$ \}. Follow up after eight months showed no evidence of recurrence.

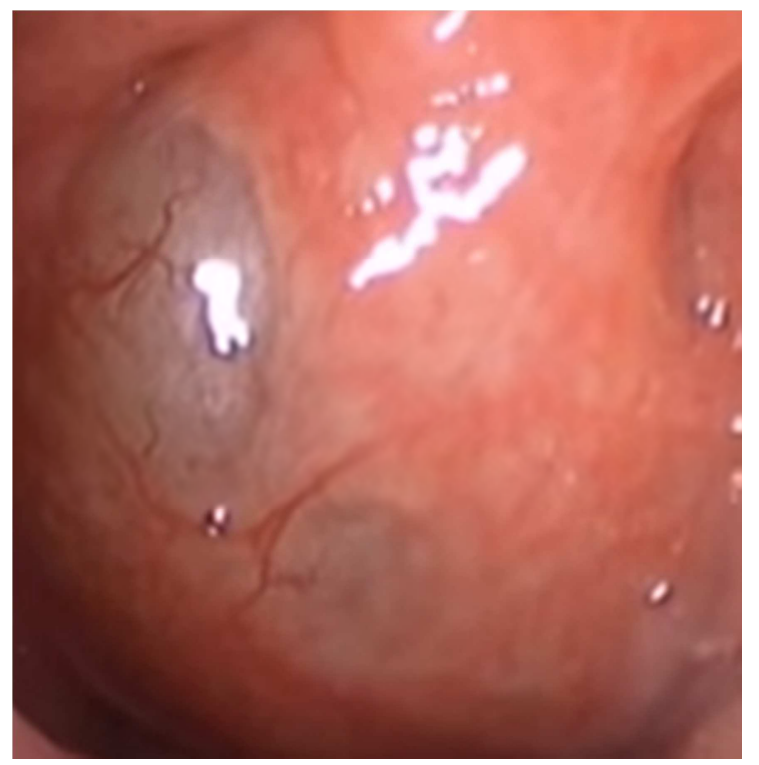

Figure 1. Nasopharyngeal cyst.

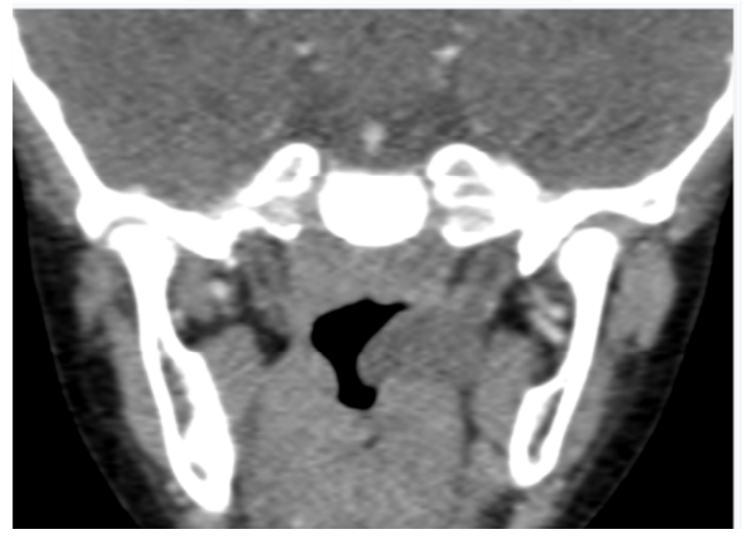

Figure 2. CT scan coronal cut show soft tissue density with hypodense area in nasopharynx with extension to oropharynx.

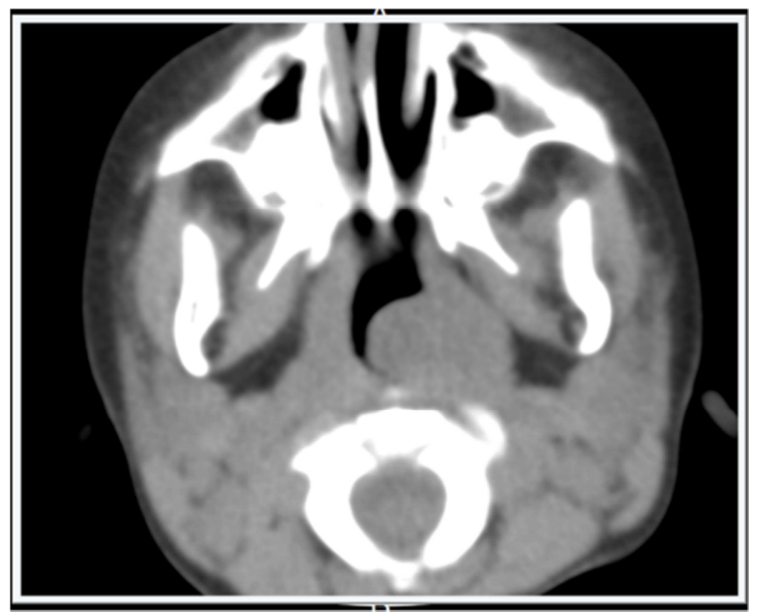

Figure 3. CT scan axial cuts showed with soft tissue density in nasopharynx.

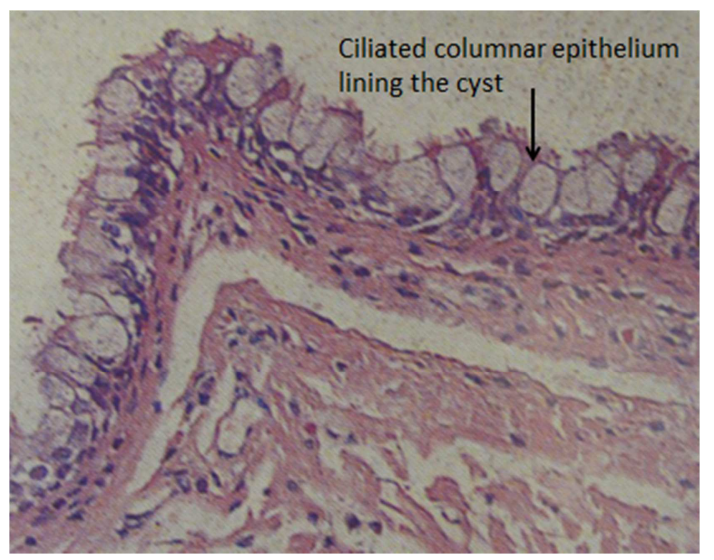

Figure 4. Branchial cleft cyst wall lined with respiratory epithelium.

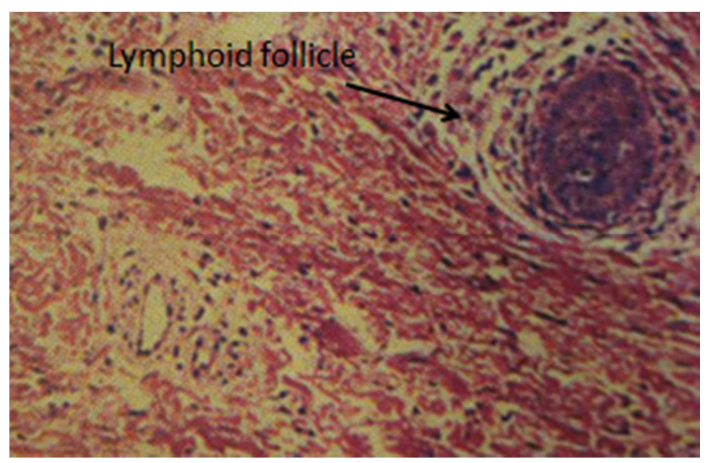

Figure 5. The branchial cyst wall containing a nodular lymphoid infiltrate.

\section{Discussion}

Branchial cleft cysts are congenital epithelial cysts, which arise on the lateral part of the neck from a failure of obliteration of the second branchial cleft in embryonic development [7]. At the fourth week of embryonic life, the development of 4 branchial (or pharyngeal) clefts results in 5 ridges known as the branchial (or pharyngeal) arches, which contribute to the formation of various structures of the head, the neck, and the thorax. The second arch grows caudally and, ultimately, covers the third and fourth arches. The buried clefts become ectoderm-lined cavities, which normally involute around week 7 of development. If a portion of the cleft fails to involute completely, the entrapped remnant forms an epithelium-lined cyst with or without a sinus tract to the overlying skin [8-11]. First BAs probably make up less than $1 \%$ of all BAs, although it has been as common as $25 \%$ of BAs in one series [12]. First BAs usually appear on the face or are related to the auricle. Work (13) described type I and type II anomalies. Type I first BAs contain only epidermoid elements without cartilage or adnexal structures. They appear as duplication anomalies of the external canal and may pass close to the facial nerve. Type II first BAs are more common. They contain both ectoderm and mesoderm and may be found in the neck [13]. Type II anomalies typically are seen after infection as an abscess below the angle of the mandible. These pass upward through the parotid gland, passing lateral or medial to the facial nerve, and end inferior to the external auditory canal or in the canal at the bony cartilaginous junction. 
Second branchial cysts are the most common type. They can be seen as a cyst, sinus, or fistula. If the membrane separating the second cleft and pouch breaks down, a complete fistulous tract may persist. Sinus tracts may otherwise occur, opening internally or externally. Second branchial cysts appear anterior to the sternomastoid muscle. The tract passes deep to second arch structures, including the external carotid artery, the stylohyoid muscle, and the posterior belly of the digastric muscle, and superficial to structures of third-arch derivation, such as the internalcarotid artery. The tract ends in the tonsillar fossa. Second branchial cysts appear as painless fluctuant masses below the angle of the mandible and anterior to the anterior border of the sternocleidomastoid muscle. These can suddenly enlarge after an upper respiratory tract infection. Although commonly first seen in children and young adults, branchial cleft cysts can first come to clinical attention at any age. Treatment is by antimicrobial therapy and surgical excision. Third branchial defects are rare. They are found lower in the neck, also anterior to the sternomastoid muscle. Third branchial cysts are deep to the third-arch derivatives, such as the glossopharyngeal nerve and the internal carotid artery, but superficial to structures of fourth-arch derivation, such as the vagus nerve. They enter the pharynx at the thyrohyoid membrane or piriform sinus. An anomaly of the fourth branchial arch was first reported by Sanborn [14] in 1972. CT scanning and MR imaging are preferred when the lesion is extensive or when it crosses multiple anatomic spaces $[15,16]$. The choice of imaging technique depends on regional preferences; It reliably confirms the cystic nature of the mass and more precisely defines the extent of the lesion and its relationship to the surrounding structures. It is also believed that all the clinically relevant information is available as clearly on CT scans as on MRI but with lower costs and with an easier imaging process [17]. Accordingly, we performed CT imaging study for our patient due to the deep anatomical nature of the mass.

Since that time, more than 60 cases have been reported; all but three have been left-sided. Fourth-arch defects arise from the apex of the piriform sinus and course inferior to the superior laryngeal nerve. They may appear as recurrent thyroiditis or recurrent lower neck abscesses [18]. Elective surgical excision has been the treatment of choice for all branchial cysts. Abscesses should first be treated with incision and drainage. The cyst should then be removed, with its tract traced to its origin in the aerodigestive system. Inspection of the piriform sinus should precede surgical exploration in third and fourth branchial cysts. Surgery with complete excision of the cyst and its neck component is the operation of choice [19]

Nasopharynx is a rare site of presentations for branchial cleft cyst. Other rare sites have been reported include paraphryngeal space and even posterior neck. Elective transoral excision under general anesthesia and after full exposure through soft palate retraction is the treatment of choice [20]. In the present case, we performed excision of the cyst via combined transoral/transnasal endoscopic approach.

\section{Conclusion}

Second branchial cleft cysts presenting in the nasopharynx are considered rare presentations of the disease. Other differential diagnosis should be always brought in mind such as mucus retention cyst, meningioencephaloceles and vascular tumors. Fiberoptic examination of such cases is mandatory to rule out laryngeal involvement and to predict the extension of the cyst for surgical intervention. We think that CT is an important investigation in such cases to rule out vascular masses and to exclude intracranial communication. Surgical excision through combined transoral/transnasal endoscopic including the tract ligation is the treatment of choice to prevent recurrence and to minimize the occurrence of possible secondary infection of the cyst.

\section{References}

[1] Koeller KK, Alamo L, Adair CF, et al., Congenital cystic masses of the neck: radiologicpathologic correlation. Radiographics. 1999 Jan-Feb; 19(1): 121-46.

[2] Kenealy JF, Torsiglieri AJ, Tom LW. Branchial cleft anomolies: a five-year retrospective review. Trans Penn Acad Ophthalmol Otolaryngol 1990; 42: 1022-1025.

[3] Papay FA, Kalucis C, Eliachar I, Tucker HM. Nasopharyngeal presentation of second branchial cleft cyst. Otolaryngol Head Neck Surg. 1994 Feb; 110(2): 232-4.

[4] Ostfeld EJ, Wiesel JM, Rabinson S, Auslander L. Parapharyngeal (retrostyloid)--third branchial cleft cyst. J Laryngol Otol. 1991 Sep; 105(9): 790-2.

[5] Grignon B, Pierucci F, Wayoff M, Roland J. Branchial cyst of unusual localization: report of a case and considerations on organogenesis. Morphologie. 1997 Sep; 81(254): 9-11.

[6] Chandler JR, Mitchell B. Branchial cleft cysts, sinuses and fistulas. Otolaryngol Clin North Am 1981; 13: 175.

[7] Wagner AM, Hansen RC. Neonatal skin and skin disorders. In: Schachner LA, Hansen RC, eds. Pediatric Dermatology. Vol 1. $2^{\text {nd }}$ ed. New York, NY: Churchill Livingston; 1995: 291-3.

[8] Doi O, Hutson JM, Myers NA, McKelvie PA. Branchial remnants: a review of 58 cases. J Pediatr Surg. Sep 1988; 23(9): 789-92.

[9] Little JW, Rickles NH. The histogenesis of the branchial cyst. Am J Pathol. 1967; 50(3): 533-47.

[10] Rickles NH, Little JW. The histogenesis of the branchial cyst. II A study of the lining epithelium. Am J Pathol. 1967; 50(5): 765-77.

[11] Telander RL, Deane SA. Thyroglossal and branchial cleft cysts and sinuses. SurgClin North Am. Aug 1977; 57(4): 779-91.

[12] Choi SS, Zalzal GH. Branchial anomolies: a review of 52 cases. Laryngoscope. 1995 Sep; 105(9 Pt 1): 909-13.

[13] Work WP. Cysts and congenital lesions of the parotid glands. Otolaryngol Clin North Am. 1977 Jun; 10(2): 339-43.

[14] Sandborn WD, Shafer AD. A branchial cleft of fourth pouch origin. J Pediatr Surg 1972 Feb; 7(1): 82. 
[15] Joshi MJ, Provenzano MJ, Smith RJ, Sato Y, Smoker WR. The rare third branchial cleft cyst. AJNR Am J Neuroradiol. 2009 Oct; 30(9): 1804-6.

[16] Koch BL. Cystic malformations of the neck in children. Pediatr Radiol 2005; 35: 463-77.

[17] Panchbhai AS, Choudhary MS. Branchial cleft cyst at an unusual location: a rare case with a brief review. Dentomaxillofac Radiol. 2012 Dec; 41(8): 696-702.
[18] Jeyakumar A, Hengerer AS. Various presentations of fourth branchial anomolies. Ear Nose Throat J. 2004 Sep; 83(9): 640-2,644.

[19] Kotecha V, Muturi A, Ruturi J. Branchial cysts: an unusual cause of a mediastinal mass: a case report. J Med Case Rep. 2015 Sep 29; 9: 208. doi: 10.1186/s13256-015-0680-y.

[20] Fageeh NA, Etwadi H, Alqarni M, Alsharif S. Nasopharyngeal branchial cleft cyst. National Journal of Otorhinolaryngology and Head \& Neck Surgery, 2015, 3(12): 30-31. 